\title{
Observability of Path Loss Parameters in WLAN-Based Simultaneous Localization and Mapping
}

\author{
Luigi Bruno and Patrick Robertson \\ Institute for Communications and Navigation \\ German Aerospace Center (DLR) \\ Wessling, Germany \\ Email: \{luigi.bruno,patrick.robertson $\} @$ dlr.de
}

\begin{abstract}
Indoor positioning by means of received signal strengths has been gathering much interest since the massive presence of wireless local area networks (WLANs) in buildings. Theoretical approaches rely on the perfect knowledge of the APs' positions and propagation conditions; since this is unrealistic in real world, we estimate such knowledge as well as the building map from data by applying Simultaneous Localization and Mapping (SLAM).

In this paper we address the joint estimation of the path loss parameters, namely the transmitted power and the path loss exponent, this latter being usually approximated in the literature by the free space value. We provide examples that show the relevance of estimating both parameters and analyze observability issues from the point of view of estimation theory. The integration of the parameter estimation in a WLAN based SLAM algorithm - WiSLAM - has been carried out and the results are discussed.
\end{abstract}

\section{INTRODUCTION}

Indoor navigation and other Location Based Services in buildings and urban areas have prompted growing demand for reliable indoor localization techniques [1], [2]. Designs of such systems should take into account features which are valuable in the context of market application, such as the cost for the user and integration of sensors in everyday objects, as well as privacy issues. Therefore, attention has been recently gathered by such sensors which are already available in a mobile phone, like accelerometers, compass, magnetometers, and radio receivers, whose data can be fused in a probabilistic framework to perform accurate positioning [3].

Information about the topology and any feature of interest of indoor environments turns out to be extremely valuable; for example, inertial navigation by means of inertial measurement units (IMUs), that are usually affected by a heavy drift in the heading error, can be made robust by exploiting the floor plan [4]-[6]. Furthermore, when radio receivers are employed, information about the transmitters and the radio propagation are needed [7].

Environmental information is easily available during supervised experiments, but cannot be assumed for wide-scale global deployments of localization techniques. One possibility is to acquire it from data by applying the Simultaneous Localization and Mapping (SLAM) approach [8]. FastSLAM is a

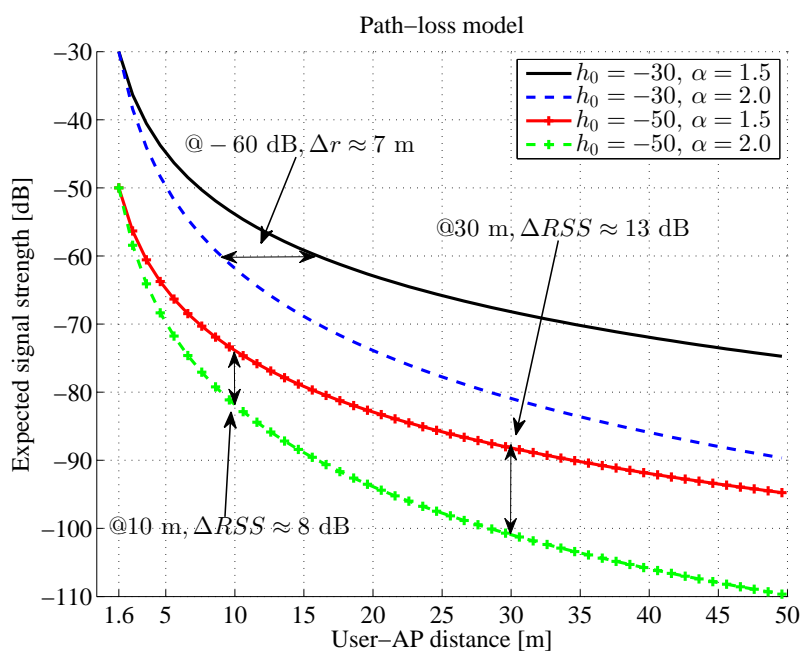

Fig. 1. Path loss model: expected RSS as a function of the user-AP distance for four couples of values $\left\{h_{0}, \alpha\right\}$.

Bayesian approach for SLAM based on the Rao-Blackwellized particle filter (RBPF) that has been applied with success to many scenarios. The user's trajectory is sampled independently for each particle, and the environmental information, or map, is then inferred for each trajectory hypothesis [9], [10]. FootSLAM uses FastSLAM approach to localize pedestrians inside buildings and at the same time to infer the building topologic map by using inertial measurements derived from a foot-mounted IMU [11], [12]; this algorithm makes use of only a local sensor and does not require any dedicated infrastructure.

Based on FootSLAM, in a previous paper we proposed WiSLAM - a Bayesian SLAM algorithm which fuses received signal strengths (RSSs) in wireless local networks (WLANs) with IMU's inertial measurements [13]. The algorithm does not assume any initial information about the position of the APs; in each particle the user's trajectory is sampled sequentially and the APs are tracked consequently, by multiplying the RSS likelihood functions. This way, a probability distribution for the AP's position is obtained, which shrinks in time 
around the right position. The evaluation of the RSS likelihood function requires the characterization of radio propagation and this is done according to the path loss model, whose parameters can actually be tuned in order to add flexibility to the algorithm [14]. In WiSLAM, the transmitted power is added to the state space and estimated by means of the RBPF, while the path loss exponent, which describes the decay of power with distance, is fixed at the free space value.

Another SLAM approach based on RSS measurements involves a representation of the radio signal across the indoor area in terms of Gaussian process latent variable models (GPLVM) [15]. However, as acknowledged by the authors, in the absence of other sensors, quite strong assumptions must be made about the user's trajectory and data association. A Graph-SLAM like approach is instead exploited in [16], where also the necessity of IMU data is stated in order to overcome limitations imposed on the user's movement.

The impact of a mismatch in the path loss exponent has been explored in several papers. An empirical study based on RSS measurements in a IEEE 802.11.b network, proposed in [17], besides validating the path loss model with additive Gaussian noise in an indoor scenario, shows the negative effects of a wrong exponent on localization performance. The more general context of wireless sensor networks is used in [18] to develop a RSS-based localization algorithm in which the path loss exponent is considered unknown. The authors combine the range estimations which are yielded by the RSS by using a spring-relaxation method: each AP-user distance is modeled by a spring, whose elasticity coefficient is made variable with distance in order to mitigate the path loss exponent inaccuracy. Their claim is that such a mismatch yields an error in the distance determination that is proportional to the distance itself; therefore, their solution is, in practice, to increase the variance of weak RSS measurements so that it includes the error yielded by the parameter mismatch. A theoretical analysis of the joint estimation of user's position and path loss exponent is instead presented in [19] based on the CramerRao Lower Bound (CRLB); the author focuses mainly on the geometry impact on the estimation performance.

Joint estimation of both transmitted power and path loss exponent is instead proposed in [20]; in that paper, authors perform a training stage to provide a maximum likelihood estimation of the parameters and then use Bayesian positioning algorithms, which account also for the residual uncertainty of parameters. Real world experiments show once again the impact of parameter estimation on localization.

Our paper, instead, proposes a SLAM Bayesian algorithm which includes the estimation of both path loss parameters, AP's positions and the user's trajectory without any calibration phase or prior information on the WLAN. In the first part of the paper we analyze the observability issues arising from the joint estimation of transmitted power and path loss exponent by means of a theoretical framework based on estimation theory. To do so, we approach the parameter estimation alone by providing the CRLB for the joint estimation and the efficient estimator, i.e. the unbiased estimator whose Mean
Squared Error (MSE) achieves the optimal value indicated by the CRLB. We discuss the geometrical conditions which are favorable to parameter observability and the impact of measurement noise. In the second part of the paper we apply estimation of both parameters to the SLAM case: we extend the WiSLAM algorithm in order to account for both parameters and we show the results of both simulations and of datasets collected in two different buildings.

The paper is structured as follows: in Section II we analyze the impact of mismatches in the path loss parameters on distances determination and, therefore, on positioning; observability of parameters in a supervised context and their estimation is instead subject of Section III; application of parameter estimation to SLAM is proposed in Section IV and the results are discussed in terms of simulations and real world experiments in Sections VI and VII, respectively; concluding remarks are given in Section VIII.

\section{Model SENSITIVITY TO PARAMETERS}

The path loss model describes in a compact but effective way the power loss due to radio propagation. It is derived from the Friis formula and states that the power $P(r)$ received at distance $r$ from the transmitter is given by [14]

$$
P(r)=P_{0}\left(\frac{d_{0}}{r}\right)^{\alpha},
$$

where $d_{0}$ is a reference distance, $P_{0}$ is a constant representing the transmitted power and antenna gains and $\alpha$ is the path loss exponent ( $\alpha=2$ in free space). This model is intended to work only in far field condition, as for $r \rightarrow 0, P(r) \rightarrow \infty$. In this paper, we use $d_{0}$ to determine the limit between near and far field, so that we will always assume $r \geq d_{0}$. Restating equation (1) in $\mathrm{dB}$ for the signal strength $h(r)$ (square root of power) we find:

$$
h(r)=h_{0}-20 \alpha \log _{10}\left(r / d_{0}\right) .
$$

In this Section we do not consider any measurement noise, since we analyze the sensitivity of the model with regards to the transmitted signal strength $h_{0}$ and the path loss exponent $\alpha$. Figure 1 shows the expected RSS with 4 different combinations of parameters. In particular, the $20 \mathrm{~dB}$ variation in $h_{0}$ is likely when different receivers are adopted; furthermore we address the case with $\alpha=2$, that is the free space value, often used even in indoor positioning, and $\alpha=1.5$ which is referred to in the literature as a likely value in indoor environments, especially in absence of obstacles between transmitter and receiver, i.e. line of sight (LOS) propagation [14].

Visual inspection of the curves in Fig. 1 shows that a variation in $h_{0}$ yields simply a shift in terms of expected RSS; a variation in the path loss exponent $\Delta \alpha$ yields a variation in the expected RSS which is proportional to the distance and assumes relevant values even at close distances: in this example, with $\Delta \alpha=0.5$, the expected RSS differs by $8 \mathrm{~dB}$ at a distance of 10 meters and by $13 \mathrm{~dB}$ at 30 meters. Inverting the perspective, the same RSS can refer to very differing distances $r$ : in the example highlighted in Fig. $1, \Delta \alpha=0.5$ 


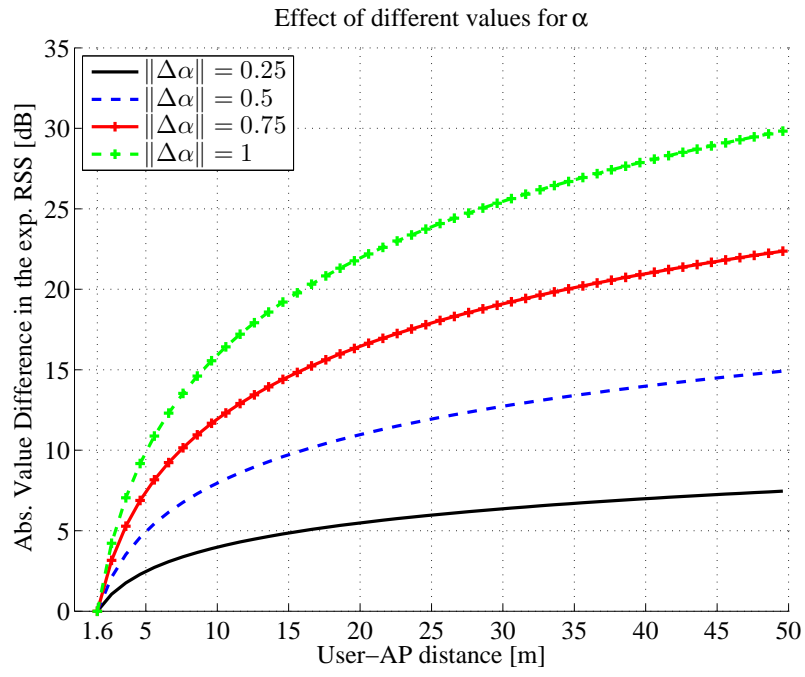

Fig. 2. Impact of mismatch $\|\Delta \alpha\|$ on the path loss exponent on the expected RSS (in absolute value) for 4 values in the range $\|\Delta \alpha\| \in[0.25,1]$.

yields about 7 meters of distance variation in the distance when the RSS is $-60 \mathrm{~dB}\left(h_{0}=-30 \mathrm{~dB}\right)$.

The absolute value of the difference in the expected RSS obtained using several values $\|\Delta \alpha\|$ up to 1 is depicted in Fig. 2 against the distance; it is linear with $\|\Delta \alpha\|$ and can assume values of tens of $\mathrm{dB}$ even at quite short distances.

\section{OBSERVABILITY OF THE PATH LOSS PARAMETERS}

We investigate the joint estimation of the path loss parameters by resorting to estimation theory. To do so, we need to introduce some more formalism.

A pedestrian walks across the area, where an arbitrary number of APs is deployed, carrying a WLAN receiver. RSS vectors are collected at a constant pace $\tau$ and they are assumed independent in time given the user's and the APs' position; furthermore we also assume that RSS from different APs are conditionally independent. From now on the time will be considered discrete, by denoting the sampling instants with the index $k=1,2, \ldots$. If $\mathbf{x}_{k}$ is the pedestrian's position at the instant $k$, the RSS measurement $y_{k}$ in $\mathrm{dB}$ from one generic AP is assumed a random variable with Gaussian distribution

$$
y_{k} \sim \mathcal{N}\left(h\left(r_{k}\right), \sigma^{2}\right),
$$

where $r_{k}=\left\|\mathbf{x}_{k}-\mathbf{x}_{A P}\right\|$ is the user-AP distance, $h\left(r_{k}\right)$ is the expected RSS given by the path loss model in (2) and $\sigma^{2}$ is the noise variance, independent of the parameters and supposed known throughout the paper. We notice that the parameters $h_{0}$ and $\alpha$ of the path loss model are present only in the mean of the measurements.

\section{A. Estimation of the parameters in absence of noise}

We now propose considerations about parameter estimation starting from a supervised set-up. Let the pedestrian position $\mathbf{x}_{k}$ be known, as well as the position of an AP $\mathbf{x}_{A P}$. By denoting for simplicity

$$
r_{k}^{\prime}=20 \log _{10}\left(\left\|\mathbf{x}_{k}-\mathbf{x}_{A P}\right\| / d_{0}\right),
$$

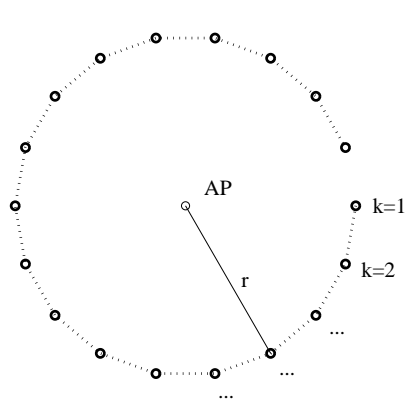

(a)

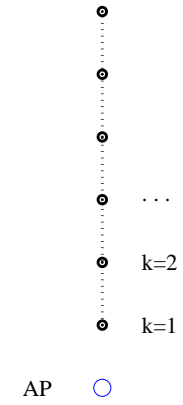

(b)
Fig. 3. Geometrical primitives: the user walks on (a) a circle centered in the $\mathrm{AP}$, (b) a line radially directed w.r.t. the AP.

we can rewrite (2) as a linear function

$$
h\left(\left\|\mathbf{x}_{k}-\mathbf{x}_{A P}\right\|\right) \rightarrow h\left(r_{k}^{\prime}\right)=h_{0}-\alpha r_{k}^{\prime},
$$

which highlights the different roles of the parameters in the path loss model.

We now show two examples with opposite results regarding observability, where, for simplicity, we neglect the measurement noise, i.e. $\sigma=0$. In the framework of Fig. 3, panel (a), the user's path lies on a circle and a number K of RSS are collected from the AP in the center. In this case the user-AP distance is constant, whence $r_{k}^{\prime} \rightarrow r^{\prime}$ and $h\left(r_{k}^{\prime}\right) \rightarrow h\left(r^{\prime}\right)$, the system of $\mathrm{K}$ equations (2), one per measurement, collapses into one independent linear equation, that is not sufficient to observe the parameters. In the second example, depicted in the framework of Fig. 3, panel (b), the user walks straightly away from the AP, and thus each measurement is collected at a different distance, i.e. $r_{k}^{\prime} \neq r_{j}^{\prime}$ if $k \neq j$. In this case an arbitrary pair of measurements can be used to evaluate the parameters, since, in absence of noise, it yields a linear system of two equation and two variables, i.e. for $k \neq j$ :

$$
h_{0}-\alpha r_{k}^{\prime}=y_{k}, h_{0}-\alpha r_{j}^{\prime}=y_{j},
$$

which admits one solution for $h_{0}$ and $\alpha$ :

$$
\alpha=\frac{y_{k}-y_{j}}{r_{j}^{\prime}-r_{k}^{\prime}}, h_{0}=y_{k}+r_{k}^{\prime} \frac{y_{k}-y_{j}}{r_{j}^{\prime}-r_{k}^{\prime}} .
$$

A plain explanation for both cases involves the model in Fig. 1 and is based on the consideration that it should be sampled at two different points at least, i.e. two measurements at different distances need to be collected, in order to specify the representation.

\section{B. CRLB in presence of Gaussian noise}

When measurement noise is considered, i.e. $\sigma>0$, the observability problem can be afforded from a probabilistic point of view. We define that a vector of parameters is observable from a set of data when a consistent estimator exists, i.e. an estimator that tends to no bias and zero variance when the size of data tends to infinity.

We claim that, adopting the model (2) with additive Gaussian measurement noise, given user's trajectory and AP's 
position, observability of $h_{0}$ and $\alpha$ is guaranteed if relevant changes in the user-AP distance are provided.

To prove our statement we first compute the Fisher Information Matrix (FIM) and the Cramer-Rao Lower Bound (CRLB) for the parameters and then provide the unbiased estimators which achieve the bound [21]. Define the parameters vector

$$
\boldsymbol{\theta}=\left[h_{0}, \alpha\right]^{T},
$$

and consider one measurement, $y_{1}$, whose distribution (3) will be denoted by $p\left(y_{1} ; \boldsymbol{\theta}\right)$, with $\mathbf{x}_{1}$ and $\mathbf{x}_{A P}$ known as before; the log-likelihood function of data is, recalling (3) and (2),

$$
\log p\left(y_{1} ; \boldsymbol{\theta}\right)=c+-\frac{1}{2 \sigma^{2}}\left(y_{1}-h_{0}+\alpha r_{1}^{\prime}\right)^{2},
$$

where $c$ is a constant, and the FIM of the parameters based on $y_{1}$ results straightforwardly following [21]

$$
\mathcal{I}_{1}(\boldsymbol{\theta})=\sigma^{-2}\left[\begin{array}{cc}
1 & -r_{1}^{\prime} \\
-r_{1}^{\prime} & \left(r_{1}^{\prime}\right)^{2}
\end{array}\right]
$$

Since the CRLB is obtained from the inverse of the FIM, we notice that the determinant is zero, yielding undefined CRLB: one measurement alone does not provide any information useful to estimate the parameters. In the case of $K$ independent measurements $\left\{y_{k}\right\}$, whose log-likelihood function is

$$
\log p\left(y_{1}, \ldots, y_{K} ; \boldsymbol{\theta}\right)=K c-\frac{1}{2 \sigma^{2}} \sum_{k=1}^{K}\left(y_{k}-h_{0}+\alpha r_{k}^{\prime}\right)^{2},
$$

the total FIM $\mathcal{I}_{K}$ is the sum of the single ones:

$$
\mathcal{I}_{K}(\boldsymbol{\theta})=\sigma^{-2}\left[\begin{array}{cc}
K & -\sum_{k=1}^{K} r_{k}^{\prime} \\
-\sum_{k=1}^{K} r_{k}^{\prime} & \sum_{k=1}^{K}\left(r_{k}^{\prime}\right)^{2}
\end{array}\right],
$$

whose determinant, semi-definite positive, results in

$$
\begin{aligned}
\operatorname{det}\left(\mathcal{I}_{K}(\boldsymbol{\theta})\right) & =\sigma^{-4}\left(K \sum_{k=1}^{K}\left(r_{k}^{\prime}\right)^{2}-\left(\sum_{k=1}^{K} r_{k}^{\prime}\right)^{2}\right) \\
& =\sigma^{-4} K^{2}\left(\frac{\sum_{k=1}^{K}\left(r_{k}^{\prime}\right)^{2}}{K}-\left(\frac{\sum_{k=1}^{K} r_{k}^{\prime}}{K}\right)^{2}\right) .
\end{aligned}
$$

In the second line of (10) we have isolated the difference between the mean square value of the $r_{k}^{\prime} \mathrm{s}$ and their squared mean. Realizing that the square function is convex, the application of the Jensen inequality yields that not only the determinant of $\mathcal{I}_{K}(\boldsymbol{\theta})$ is non negative, in accordance to the FIM definition, but also that it is zero if and only if all terms $r_{k}^{\prime}$ are equal [21]. Conversely, when terms $r_{k}^{\prime}$ are not equal, $\operatorname{det}\left(\mathcal{I}_{K}(\boldsymbol{\theta})\right)>0$, and the CRLB matrix

$$
\mathcal{C}_{K}(\boldsymbol{\theta})=\left(\mathcal{I}_{K}(\boldsymbol{\theta})\right)^{-1}
$$

is well defined. The diagonal terms of the CRLB matrix, henceforth denoted by $\mathcal{C}_{K}\left(h_{0}\right)$ and $\mathcal{C}_{K}(\alpha)$ respectively, provide lower bounds to the variance achieved by any corresponding unbiased estimator. Therefore we find for any unbiased estimator $\hat{h}_{0}$ of the transmitted power:

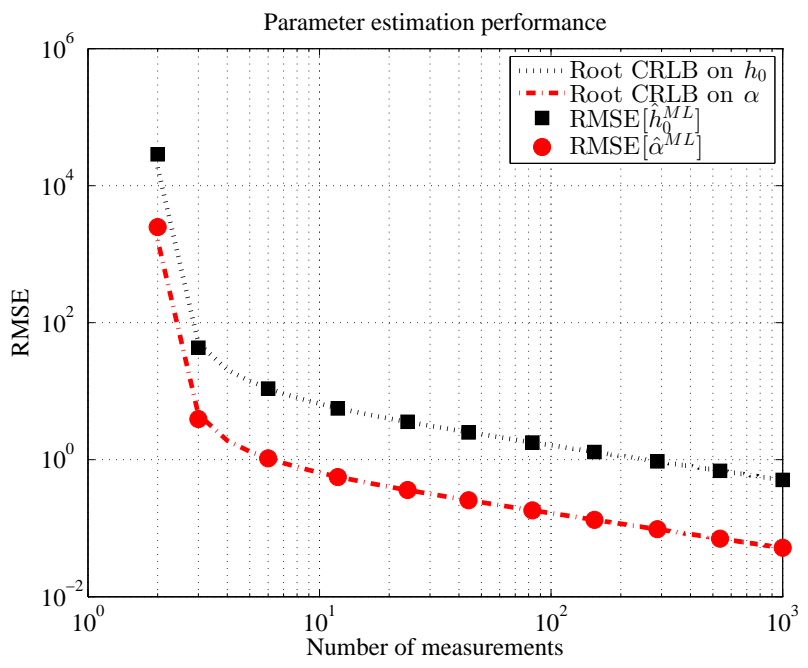

Fig. 4. Performance of the joint unbiased estimators for $h_{0}$ and $\alpha$ : root CRLB (dotted lines) and root mean square error (RMSE) computed for some sizes of the dataset (markers).

$$
\begin{aligned}
\operatorname{VAR}\left[\hat{h_{0}}\right] \geq \mathcal{C}_{K}\left(h_{0}\right) & =\frac{\sigma^{-2} \sum_{k=1}^{K}\left(r_{k}^{\prime}\right)^{2}}{\operatorname{det}\left(I_{K}\right)} \\
& =\frac{\sigma^{2}}{K} \frac{\left(\sum_{k=1}^{K}\left(r_{k}^{\prime}\right)^{2}\right) / K}{\frac{\sum_{k=1}^{K}\left(r_{k}^{\prime}\right)^{2}}{K}-\left(\frac{\sum_{k=1}^{K} r_{k}^{\prime}}{K}\right)^{2}},
\end{aligned}
$$

and for any unbiased estimator $\hat{\alpha}$ of the path loss exponent:

$$
\begin{aligned}
\operatorname{VAR}[\hat{\alpha}] \geq \mathcal{C}_{K}(\alpha) & =\frac{\sigma^{-2} K}{\operatorname{det}\left(I_{K}\right)} \\
& =\frac{\sigma^{2}}{K} \frac{1}{\frac{\sum_{k=1}^{K}\left(r_{k}^{\prime}\right)^{2}}{K}-\left(\frac{\sum_{k=1}^{K} r_{k}^{\prime}}{K}\right)^{2}} .
\end{aligned}
$$

Both bounds tend asymptotically to zero if $r_{k}^{\prime}$ is assumed limited, so that the difference between mean square value and square mean cannot diverge. This condition is widely accomplished, since the far field condition yields $r_{k}^{\prime} \geq 0$ and the maximum range achieved by an AP is no more than $100 \mathrm{~m}$ $\left(r_{k}^{\prime} \leq 20 \log _{10}(100 / 1.6) \approx 35.9\right)$, becoming lower in practical scenarios. Finally, we can notice in the expressions of both bounds the absence of the true parameters.

In Fig. 4 the root CRLBs for both estimators are denoted by dashed lines as functions of the numbers of measurements up to $N=1000$. The results have been obtained in the linear scenario of Fig. 3, panel (b), with $\sigma=3$, by sampling the userAP distances independently between 1.6 and 50 meters and then averaging on $10^{4}$ trajectories. The asymptotic behavior of the root CRLB, proportional to $1 / \sqrt{N}$, is evident already after as few as 10 measurements; the two orders of magnitude between the CRLBs in favor of $\alpha$ are reasonable, since the path loss exponent has a smaller variation range than $h_{0}$.

\section{MMSE unbiased estimator}

We now show that the maximum likelihood (ML) estimators for $h_{0}$ and $\alpha$ are unbiased and achieve the CRLB, which ensures their optimality in the minimum mean square error (MMSE) sense [21]. By derivating the log-likelihood function 
of data in (9) with regards to both parameters, we get the system of equations:

$$
\begin{aligned}
& \sum_{k=1}^{N}\left(y_{k}-h_{0}+\alpha r_{k}^{\prime}\right)=0 \\
& \sum_{k=1}^{N} r_{k}^{\prime}\left(y_{k}-h_{0}+\alpha r_{k}^{\prime}\right)=0,
\end{aligned}
$$

whose solution provides the ML estimator for $\alpha$ :

$$
\hat{\alpha}^{M L}=\frac{\sum_{k=1}^{N} r_{k}^{\prime} y_{k}-\frac{1}{N} \sum_{k=1}^{N} y_{k} \sum_{k=1}^{N} r_{k}^{\prime}}{\frac{1}{N}\left(\sum_{k=1}^{N} r_{k}^{\prime}\right)^{2}-\sum_{k=1}^{N}\left(r_{k}^{\prime}\right)^{2}}
$$

and, consequently, for $h_{0}$ :

$$
\hat{h}_{0}^{M L}=\frac{1}{N} \sum_{k=1}^{N} y_{k}+\frac{\hat{\alpha}^{M L}}{N} \sum_{k=1}^{N} r_{k}^{\prime} .
$$

applying $\mathrm{E}\left[y_{k}\right]=h_{0}-\alpha r_{k}^{\prime}$ and $\operatorname{VAR}\left[y_{k}\right]=\sigma^{2}$, as well as independence between measurements, we show that

$$
\mathrm{E}\left[\hat{h}_{0}^{M L}\right]=h_{0}, \mathrm{E}\left[\hat{\alpha}^{M L}\right]=\alpha,
$$

and, moreover, their variances attain the corresponding CRLBs. This shows that ML estimators are optimal in the MMSE sense, at least in the class of unbiased estimators, and proves the observability of the path loss parameters when user's trajectory and AP position are known.

Our result is validated by simulations, whose results are denoted by the curves with markers in Fig. 4. We compare the root MSE (RMSE) obtained by the ML estimators of the parameters with the root of CRLB computed in Section III-B. For each trajectory the true values of $\alpha$ and $h_{0}$ are drawn randomly in the ranges $[1,4]$ and $[-60,-30]$ respectively, the user-AP distances are those being used to compute the CRLB and again, the results are averaged over $10^{4}$ independent trials. The ML estimates are carried out for 11 values of $N_{H}$ chosen between 2 and 1000, and the theoretical performance is always achieved.

\section{ESTIMATION OF PROPAGATION PARAMETERS IN SLAM}

We address the joint estimation of transmitted power and path loss exponent in the WiSLAM framework, by augmenting the state space of the underlying RBPF. In WiSLAM the transmitted power only is estimated in a probabilistic way; we now extend the state space by adding also the path loss exponent and evaluating observability issues in this framework. Our claim is that convergence of the algorithm is not prevented by the new variable and that the observability of the path loss parameters is also preserved. We start by deriving our extended version of WiSLAM with particular attention to the novelty.

\section{A. Extended WiSLAM derivation}

WiSLAM is a Bayesian algorithm whose state space includes several variables: the user's trajectory, in terms of positions and poses, $\mathbf{P}_{0: k}$; auxiliary variables related to the exploitation of IMU measurements, i.e. the step sequence, $\mathbf{U}_{0: k}$, and step measurement process, $\mathbf{E}_{0: k}$; the maps of interest, i.e.

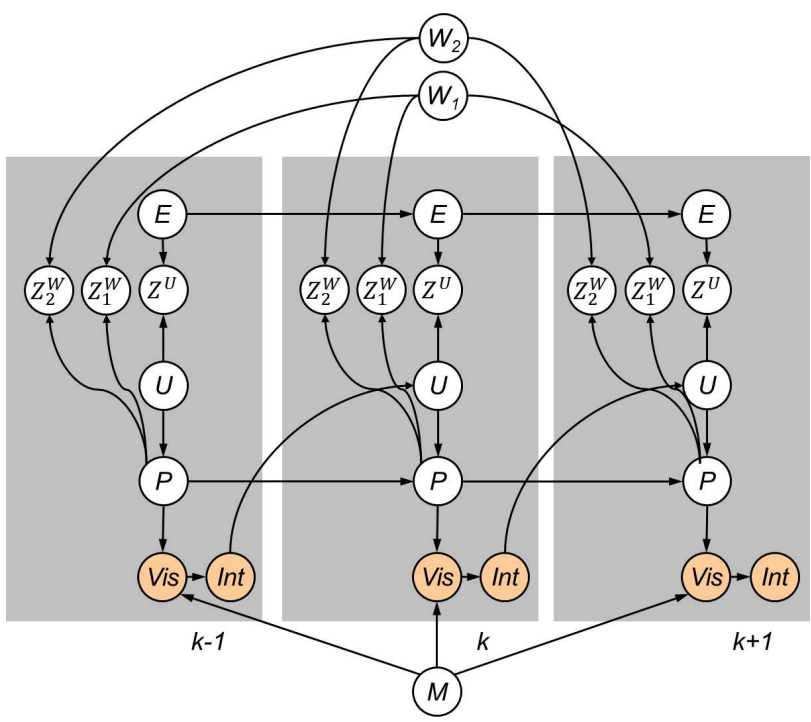

Fig. 5. The DBN following [11]-[13] for our scenario showing three time slices and two APs; for the meaning of the symbols refer to the main text.

the FootSLAM map, M [11], [12], and the WLAN map for each of the APs, $\mathbf{W}_{j}$ for the $\mathrm{j}$-th AP, consisting of its position and parameters. The maps are both considered stationary and initially unknown (uninformative prior). The measurements are of two types: step measurements, $\mathbf{Z}_{k}^{U}$ and RSS measurements $\mathbf{Z}_{k}^{W}$ in $\mathrm{dB}$.

The relations among the variables are encoded in the Dynamic Bayesian Network (DBN) of Fig. 5, in which intention Int and visual system Vis of the pedestrian drive her steps but are not observable [11]. The FootSLAM map M can include any features and information to let the pedestrian choose Int, while each WLAN map $\mathbf{W}_{j}$ contains the position and path loss parameters of the corresponding AP. Since the WLAN maps and measurements are conditionally independent, from now on we will consider for simplicity only one AP, omitting its index.

The goal of the algorithm is to compute the posterior pdf of the state given the measurements (and the priors when informative):

$$
p\left(\mathbf{P}_{0: k} \mathbf{U}_{0: k} \mathbf{E}_{0: k}, \mathbf{W}, \mathbf{M} \mid \mathbf{Z}_{1: k}^{U}, \mathbf{Z}_{1: k}^{W}\right),
$$

which can be factorized as follows:

$$
p\left(\mathbf{M} \mid \mathbf{P}_{0: k}\right) \cdot p\left(\mathbf{W} \mid \mathbf{P}_{0: k}, \mathbf{Z}_{1: k}^{W}\right) \cdot p\left(\mathbf{P}_{0: k} \mathbf{U}_{0: k} \mathbf{E}_{0: k} \mid \mathbf{Z}_{1: k}^{U}, \mathbf{Z}_{1: k}^{W}\right) .
$$

The first two terms relate to mapping and the last one to localization. This latter admits a recursive formulation based on the relationships encoded in the DBN:

$$
\begin{aligned}
& p\left(\{\mathbf{P U E}\}_{0: k} \mid \mathbf{Z}_{1: k}^{W}, \mathbf{Z}_{1: k}^{U}\right) \propto \\
& p\left(\mathbf{Z}_{k}^{U} \mid\{\mathbf{U E}\}_{k}\right) \cdot p\left(\mathbf{Z}_{k}^{W} \mid \mathbf{P}_{0: k}, \mathbf{Z}_{1: k-1}^{W}\right) \\
& \cdot p\left(\left\{\mathbf{E}_{k} \mid \mathbf{E}_{k-1}\right\}\right) \cdot p\left(\{\mathbf{P U}\}_{k} \mid\{\mathbf{P U}\}_{0: k-1}\right) \\
& \cdot p\left(\{\mathbf{P U E}\}_{0: k-1} \mid \mathbf{Z}_{1: k-1}^{U}, \mathbf{Z}_{1: k-1}^{W}\right) .
\end{aligned}
$$

In order to compute all the factors in (18) we need to marginalize some of the terms on the maps. The FootSLAM 
map determines the term concerning the present step and position given the past ones [11], [12]:

$$
\begin{aligned}
I_{k}^{M} & \hat{=} p\left(\{\mathbf{P U}\}_{k} \mid\{\mathbf{P} \mathbf{U}\}_{0: k-1}\right) \\
& =\int_{\mathbf{M}} p\left(\mathbf{P}_{k}, \mathbf{U}_{k} \mid \mathbf{M}, \mathbf{P}_{k-1}\right) \cdot p\left(\mathbf{M} \mid \mathbf{P}_{0: k-1}\right) d \mathbf{M} .
\end{aligned}
$$

The WLAN map determines instead the RSS likelihood function [13]:

$$
\begin{aligned}
I_{k}^{W} & \hat{=} p\left(\mathbf{Z}_{k}^{W} \mid \mathbf{P}_{0: k}, \mathbf{Z}_{1: k-1}^{W}\right) \\
& =\int_{\mathbf{W}} p\left(\mathbf{Z}_{k}^{W} \mid \mathbf{W}, \mathbf{P}_{k}\right) \cdot p\left(\mathbf{W} \mid \mathbf{P}_{0: k-1}, \mathbf{Z}_{1: k-1}^{W}\right) d \mathbf{W} .
\end{aligned}
$$

The models which are adopted to describe and devise the step measurements, $\mathbf{Z}_{k}^{U}$, from IMU raw data are proposed in the FootSLAM papers and therein references.

\section{B. WLAN map learning anf path loss parameter estimation}

The WLAN map is composed of the AP's position $\mathbf{x}_{A P}$ and the path loss parameters $h_{0}$ and $\alpha$. The map distribution (second term from left in (17)), that has an active role in (20), can be factorized as follows:

$$
\begin{aligned}
p\left(\mathbf{W} \mid \mathbf{P}_{0: k}, \mathbf{Z}_{1: k}^{W}\right)= & p\left(\mathbf{x}_{A P} \mid h_{0}, \alpha, \mathbf{P}_{0: k}, \mathbf{Z}_{1: k}^{W}\right) \\
& \cdot p\left(h_{0} \mid \alpha, \mathbf{P}_{0: k}, \mathbf{Z}_{1: k}^{W}\right) \cdot p\left(\alpha \mid \mathbf{P}_{0: k}, \mathbf{Z}_{1: k}^{W}\right) .
\end{aligned}
$$

The first two terms on the right hand side of (21) are equivalent to the ones in former WiSLAM [13], with the formal difference that here we make the dependency on $\alpha$ explicit. The distribution of the AP's position given the parameters $h_{0}$ and $\alpha$ is the product of the RSS likelihood functions, each one being a circular pdf centered on the user's position in $\mathbf{P}_{k}$

$$
\begin{aligned}
p\left(\mathbf{x}_{A P} \mid h_{0}, \alpha, \mathbf{P}_{0: k}, \mathbf{Z}_{1: k}^{W}\right) \propto & p\left(\mathbf{Z}_{k}^{W} \mid \mathbf{x}_{A P}, h_{0}, \alpha, \mathbf{P}_{k}\right) \\
& \cdot p\left(\mathbf{x}_{A P} \mid h_{0}, \alpha, \mathbf{P}_{0: k-1}, \mathbf{Z}_{1: k-1}^{W}\right) \\
& \propto \prod_{s=1}^{k} p\left(\mathbf{Z}_{s}^{W} \mid \mathbf{x}_{A P}, h_{0}, \alpha, \mathbf{P}_{s}\right) .
\end{aligned}
$$

The transmitted power is modeled as a finite discrete random variable with $2-3 \mathrm{~dB}$ spaced values $\left\{h_{h}\right\}_{h=1, \ldots, N_{H}}$. Accordingly, the statistical characterization of $h_{0}$ is provided by the probabilities of all $h_{h}$, which are computed recursively by means of the Bayes rule (uniform prior is here assumed)

$$
\begin{aligned}
& \operatorname{Pr}\left(h_{h} \mid \alpha, \mathbf{P}_{0: k}, \mathbf{Z}_{1: k}^{W}\right) \propto p\left(\mathbf{Z}_{k}^{W} \mid h_{h}, \alpha, \mathbf{P}_{0: k}, \mathbf{Z}_{1: k-1}^{W}\right) \\
& \cdot \operatorname{Pr}\left(h_{h} \mid \alpha, \mathbf{P}_{0: k-1}, \mathbf{Z}_{1: k-1}^{W}\right),
\end{aligned}
$$

and normalization. Combining (22) and (23) we obtain for the joint pdf of $\mathbf{x}_{A P}$ and $h_{0}$ a mixture of RSS likelihood products:

$$
\begin{aligned}
& p\left(\mathbf{x}_{A P}, h_{0} \mid \alpha, \mathbf{P}_{0: k}, \mathbf{Z}_{1: k}^{W}\right)= \\
& \sum_{h=1}^{N_{H}}\left\{\operatorname{Pr}\left(h_{h} \mid \alpha, \mathbf{P}_{0: k}, \mathbf{Z}_{1: k}^{W},\right)\right. \\
& \left.\quad \cdot p\left(\mathbf{x}_{A P} \mid h_{h}, \alpha, \mathbf{P}_{0: k}, \mathbf{Z}_{1: k}^{W}\right) \cdot \delta\left(h_{0}-h_{h}\right)\right\},
\end{aligned}
$$

where $\delta(\cdot)$ denotes the Dirac delta function.

To perform the estimation of $\alpha$, we use the same technique as for $h_{0}$ : we model $\alpha$ in terms of a discrete random variable with values in a finite set $\left\{\alpha_{s}\right\}_{s=1, \ldots, N_{\alpha}}$ and update recursively their probabilities by applying the Bayes rule:

$$
\begin{aligned}
\operatorname{Pr}\left(\alpha_{s} \mid \mathbf{P}_{0: k}, \mathbf{Z}_{1: k}^{W}\right) \propto p\left(\mathbf{Z}_{k}^{W} \mid \mathbf{P}_{0: k}, \mathbf{Z}_{1: k-1}^{W}\right) & \\
& \cdot \operatorname{Pr}\left(\alpha_{s} \mid \mathbf{P}_{0: k-1}, \mathbf{Z}_{1: k-1}^{W}\right),
\end{aligned}
$$

starting from a uniform prior and normalizing after each step. The final expression for the WLAN map pdf is, therefore,

$$
\begin{aligned}
& p\left(\mathbf{W} \mid \mathbf{P}_{0: k}, \mathbf{Z}_{1: k}^{W}\right)= \\
& \sum_{s=1}^{N_{\alpha}} \operatorname{Pr}\left(\alpha_{s}, \mid \mathbf{P}_{0: k}, \mathbf{Z}_{1: k}^{W},\right)\left\{\sum_{h=1}^{N_{H}} \operatorname{Pr}\left(h_{h} \mid \alpha_{s}, \mathbf{P}_{0: k}, \mathbf{Z}_{1: k}^{W},\right)\right. \\
& \left.\cdot p\left(\mathbf{x}_{A P} \mid h_{h}, \alpha_{s}, \mathbf{P}_{0: k}, \mathbf{Z}_{1: k}^{W}\right) \cdot \delta\left(h_{0}-h_{h}\right) \cdot\right\} \delta\left(\alpha-\alpha_{s}\right) .
\end{aligned}
$$

The complexity issues arising from the extra sum in (26) with respect to (24) will be discussed after implementation.

\section{PARTICle Filter ImPlementation}

Implementation is similar to WiSLAM's in [13], in which the variable $\alpha$ is embedded in the WLAN map; it resorts to the RBPF in which at time $k$ the variables $\{\mathbf{P}, \mathbf{U}, \mathbf{E}\}_{0: k}$ are sampled according to the function [9], [11]

$$
p\left(\mathbf{U}_{k} \mid \mathbf{Z}_{k}^{U}, \mathbf{E}_{k}^{i}\right) \cdot p\left(\mathbf{E}_{k} \mid \mathbf{E}_{k-1}^{i}\right),
$$

from right to left, for the $i-t h$ particle. Each map provides a multiplicative contribution to the particle weights:

$$
w_{k}^{i} \propto w_{k-1}^{i} \cdot I_{k}^{M, i} \cdot I_{k}^{W, i},
$$

where $I_{k}^{M, i}$ and $I_{k}^{W, i}$ are suitable numerical approximations for $I_{k}^{M}$ in (19) and $I_{k}^{W}$ in (20), respectively (further APs result in independent terms $I_{k}^{W, i}$ ). The approximation underneath $I_{k}^{M, i}$ resorts to the Markov Random Field built on a discretized grid and it is discussed in [11]. As for the WLAN map approximation, for any given couple of parameter values indexed by $(h, s)$, the pdf of the AP's position in (26) undergoes a Gaussian Mixture Model (GMM) approximation with fixed number of components:

$$
\begin{aligned}
& p\left(\mathbf{x}_{A P} \mid h_{h}, \alpha_{s}, \mathbf{P}_{0: k}, \mathbf{Z}_{1: k}^{W}\right) \approx \\
& \hat{p}\left(\mathbf{x}_{A P} \mid h_{h}, \alpha_{s}, \mathbf{P}_{0: k}, \mathbf{Z}_{1: k}^{W}\right)=\sum_{p=1}^{N_{\text {peaks }}} \tilde{u}_{p, k} \cdot f_{p, k}\left(\mathbf{x}_{A P}, h, s\right)
\end{aligned}
$$

where $\tilde{u}_{p, k}$ is a set of non-negative coefficients whose sum over $p$ is one and $f_{p, k}\left(\mathbf{x}_{A P}, h, s\right)$ are Gaussian distributed components. When an AP is reliably detected for the first time, the corresponding GMM (29) is initialized from the user's trajectory and the measurements according to the Maximum Likelihood criterion [21]. The procedure is repeated for all couples $(h, s)$ of parameter values; the starting probabilities for both $h_{0}$ and $\alpha$ are set to be uniform. At any new measurement from the same AP, model (29) is now updated by transforming the old GMM into a new one, since it can be seen that [13]

$$
\begin{aligned}
p & \left(\mathbf{Z}_{k+1}^{W} \mid \mathbf{x}_{A P}, h_{h}, \alpha_{s}, \mathbf{P}_{k+1}\right) \cdot \hat{p}\left(\mathbf{x}_{A P} \mid h_{h}, \alpha_{s}, \mathbf{P}_{0: k}, \mathbf{Z}_{1: k}^{W}\right) \\
& \approx \sum_{p=1}^{N_{\text {peaks }}} u_{p, k+1}(h, s) f_{p, k+1}\left(\mathbf{x}_{A P}, h, s\right)
\end{aligned}
$$




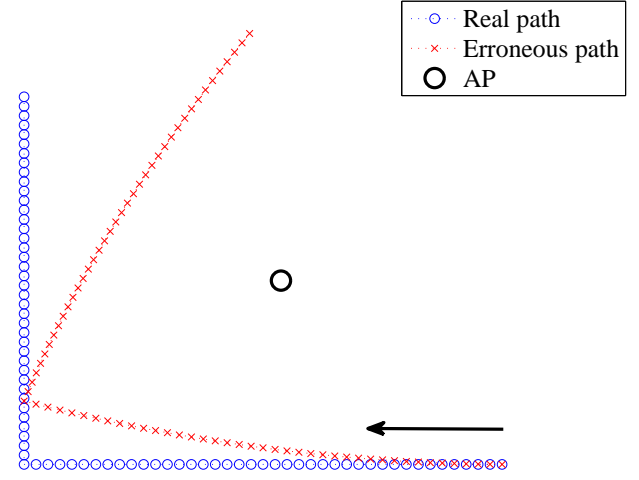

Fig. 6. Simulative scenario with the true trajectory (blue line with circles) and a corrupted one (red line with crosses); the AP is denoted by a black circle, and the measurements are collected in the points of the trajectory denoted by markers; the arrow on the lower side indicates the direction of the walk.

where the new set of coefficients and parameters of the Gaussian functions are computed by closed formula. Coefficients $u_{p, k+1}(s, h)$ should be normalized over $p$ to get the new WLAN map. However, the new probabilities of $h_{h}$ and $\alpha_{s}$ require only sums of unnormalized coefficients $u_{p, k+1}(s, h)$ w.r.t. the corresponding index. The updated $\operatorname{Pr}\left(h_{h} \mid \alpha_{s}, \mathbf{P}_{0: k+1}, \mathbf{Z}_{1: k+1}^{W}\right)$ and is proportional to (a normalization is then needed):

$$
\operatorname{Pr}\left(h_{h} \mid \alpha_{s}, \mathbf{P}_{0: k}, \mathbf{Z}_{1: k}^{W}\right) \cdot \sum_{h=1}^{N_{H}} u_{p, k+1}(h, s),
$$

while for $\alpha$ :

$\operatorname{Pr}\left(\alpha_{s} \mid \mathbf{P}_{0: k+1}, \mathbf{Z}_{1: k+1}^{W}\right) \propto \operatorname{Pr}\left(\alpha_{s} \mid \mathbf{P}_{0: k}, \mathbf{Z}_{1: k}^{W}\right) \sum_{s=1}^{N_{\alpha}} u_{p, k+1}(h, s)$.

The WLAN contribution to the weight, $I_{k}^{W, i}$, result in

$$
\begin{aligned}
I_{k}^{W, i}= & \sum_{s=1}^{N_{\alpha}} \operatorname{Pr}\left(\alpha_{s}, \mid \mathbf{P}_{0: k}, \mathbf{Z}_{1: k}^{W},\right) \\
& \cdot\left\{\sum_{h=1}^{N_{H}} \operatorname{Pr}\left(h_{h} \mid \alpha_{s}, \mathbf{P}_{0: k}, \mathbf{Z}_{1: k}^{W},\right) \cdot u_{p, k}(h, s)\right\} .
\end{aligned}
$$

We provide next a summary of extended WiSLAM.

\section{Algorithm (Extended WiSLAM).}

- Initialize all $N_{P}$ particles to $\mathbf{P}_{0}^{i}=(x, y, h=0)$ where $x, y$ and $h$ denote the pose location and heading in two dimensions; draw $\mathbf{E}_{0}^{i}$ from a suitable initial distribution for the odometry error state.

Then, for each time step increment $k$ and any particles:

- Draw $E_{k}^{i}, U_{k}^{i}$ from the proposal density in (27), compute $\mathbf{P}_{k}^{i}$ by adding the vector $\mathbf{U}_{k}^{i}$ to $\mathbf{P}_{k-1}^{i}$.

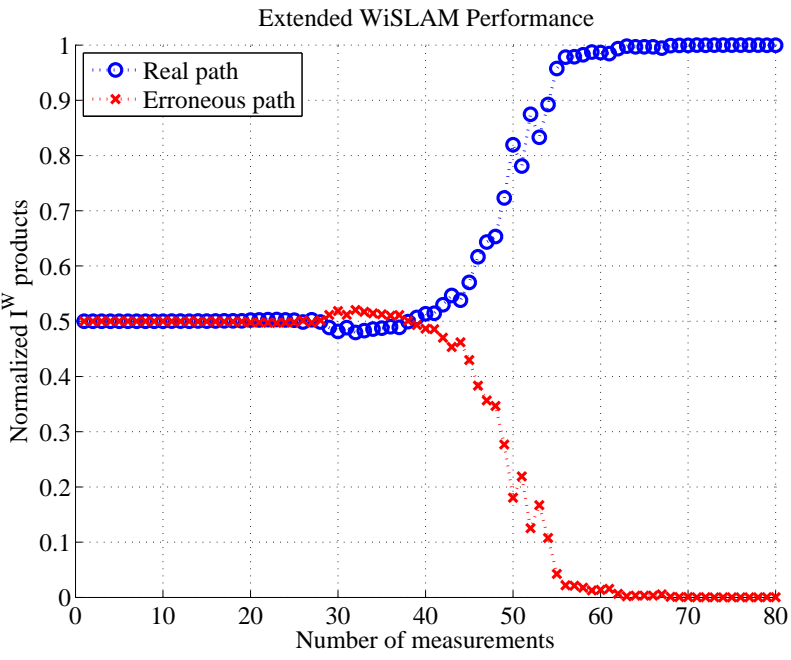

(a) Cumulative weights

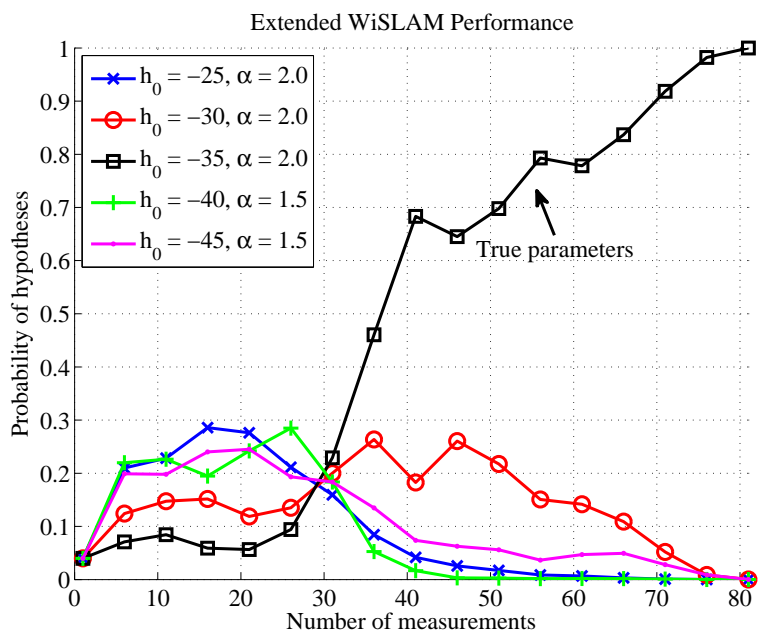

(b) Hypothesis probabilities

Fig. 7. (a) Normalized cumulative products of the WLAN weights for both particles in function of the number of RSS measurements and (b) probability of most likely hypotheses for the real trajectory in the simulative scenario depicted in Fig. 6.

- For all previously initialized APs, and all components of the corresponding GMM:

- Update peaks' parameters as in [13, Eqs. (20)-(24)].

- Compute the unnormalized $u_{p, k}(h, s)$ as in [13, Eq. (25)].

- Normalize the $u_{p, k}(h, s)$ over $p$ to obtain $\tilde{u}_{p, k}(h, s)$.

- Update $h_{0}$ and $\alpha$ distribution by means of (31) and (32), normalizing then on $h$ and $s$, respectively.

- Compute $I_{W}^{i}$ as in (33).

- Update the particle weights as in (28), where $I_{M}^{i}$ are computed as in FootSLAM [11, Eq. (5)].

- Decide if any detected but not yet employed AP should be processed and, if so, initialize new APs' posterior.

- Update the map $\mathbf{M}$ as in FootSLAM [11, Eq. (4)].

- Resampling can be performed if required. 


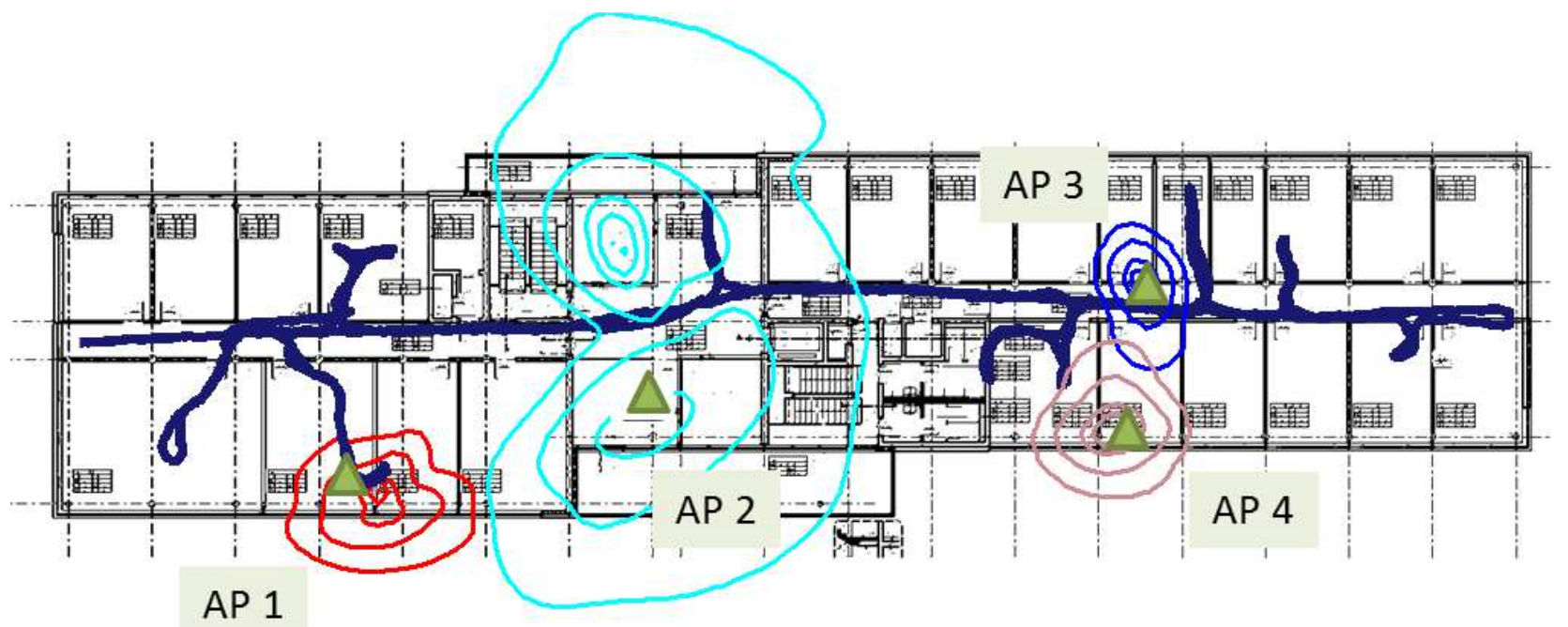

(a) Extended WiSLAM

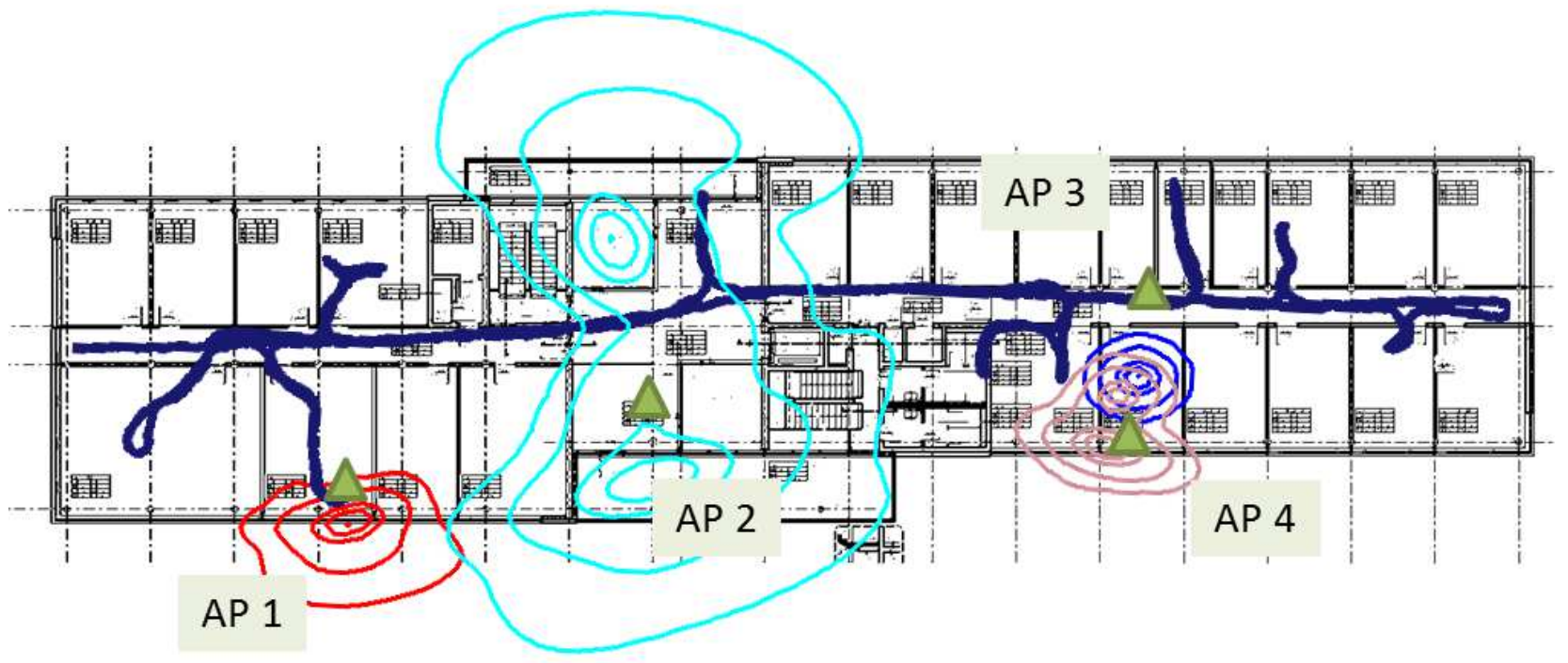

(b) Classic WiSLAM

Fig. 8. Experiments conducted in a building with 4 APs: the most weighted trajectory is denoted by a blue line and fit in the ground truth for comparison; the AP's true positions are denoted by green triangles and the corresponding pdfs by contour lines, each one denoting, from the outer to the inner the points with $1 \%, 10 \%, 50 \%, 75 \%$ and $99 \%$ of the maximal pdf value; extended WiSLAM results are in panel (a), classic WiSLAM in panel (b).

With respect to WiSLAM, additional complexity is due to the fact that more GMMs have to be trained, for each particle, to explore the $\alpha$ range of variation. The number of GMMs (29) is $N_{H} \times N_{P}$ in WiSLAM, whereas it becomes $N_{\alpha} \times N_{H} \times$ $N_{P}$ in the extended WiSLAM. As for the update step, the increase of GMMs means effort at each new measurement but the complexity is still linear in time. However, the effect of the new variable is mitigated by the restricted range of variation of $\alpha$ and by the consideration that, based on the simulation of Fig. 2, the resolution of the values $\alpha_{s}$ can be as coarse as 0.5 , since the standard deviation of noise measurements in the real world usually lies between 5 and $10 \mathrm{~dB}$.

\section{Simulative RESUlts}

Consider the example of Fig. 6 with only two idealized particles: the former follows the true trajectory of the user (blue line with circles), whereas the latter's trajectory (red with squares) is corrupted by an angular error that is quadratic in time and resembles a likely step measurement sequence. A single AP is located internally to the trajectory and 80 independent measurements are generated in $\mathrm{dB}$ according to the path loss model (2) and are corrupted by additive Gaussian noise with variance $\sigma^{2}=5$. The parameters are set to $h_{0}=-35 \mathrm{~dB}$ and $\alpha=2$ but are considered initially 


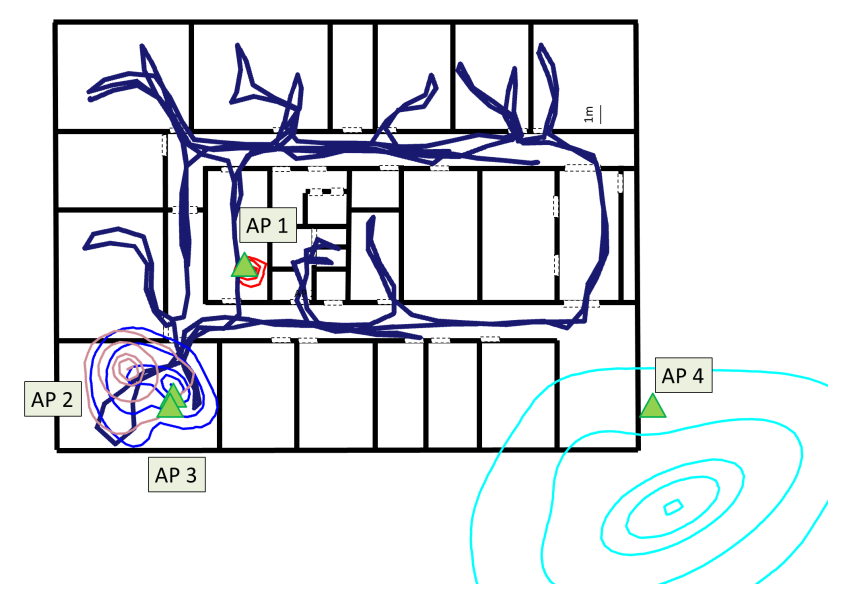

(a) Extended WiSLAM

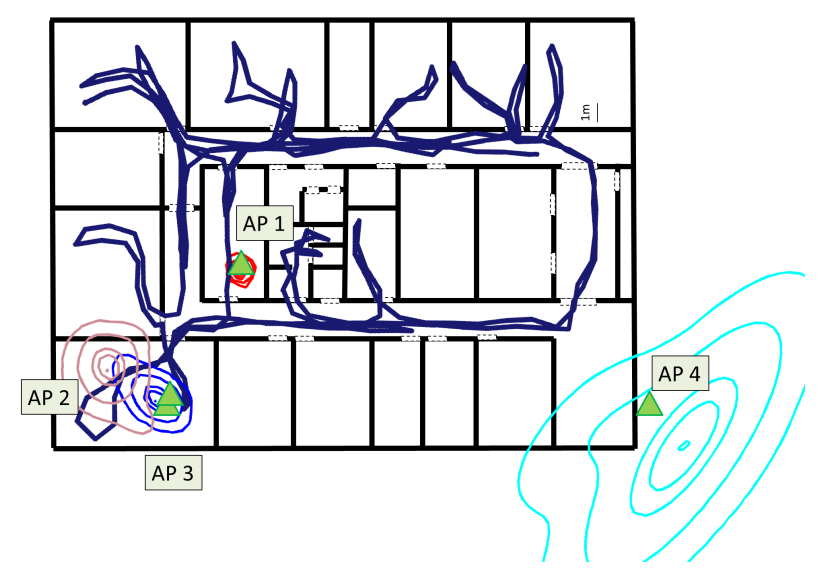

(b) Classic WiSLAM

Fig. 9. Further experimental testbed with 4 APs as well; the graphic details are the same as in Fig. 8; extended WiSLAM results are in panel (a), classic WiSLAM in panel (b).

unknown. Finally, we have repeated the simulation 10 times and we present here the averaged results.

For this example we do not resort to the GMM representation, but rather we sample the functions involved in the RBPF of Section IV, since we are using only two particles. For both parameters we allow 5 hypotheses, i.e. $N_{H}=N_{\alpha}=5$, in which the true values are included, so that the final number of hypotheses is 25 . For the transmitted power hypotheses we use a $5 \mathrm{~dB}$ step while a 0.5 step is employed for $\alpha$. We have computed the WLAN contribution (20) to the weights for both particles and all time instants: their cumulative products

$$
\prod_{k=1}^{K} I_{k}^{W, i}
$$

represent, in absence of the FootSLAM weight (19), the complete particle weights (28) and are depicted after normalization in Fig. 7, panel (a). The real trajectory attains a clear preference after about 45 measurements and the algorithm takes further 15 measurements to definitively discard the wrong path. The initial latency corresponds to the time needed by the algorithm to discriminate the right hypothesis about the path loss parameters. The probability evolutions for those hypotheses are traced in Fig. 7, panel (b); for clarity, we have selected only the hypotheses which show higher probabilities at some point, thus neglecting the ones that are never considered likely by the algorithm. The true hypothesis becomes dominant after about 40 measurements, just before the right particle starts having a significantly higher weight, as seen in Fig. 7, panel (a).

\section{REAL WORLD EXPERIMENTS}

Extended WiSLAM has been tested in two environments and compared with the case in which $\alpha=2$, henceforth called classic WiSLAM. Fig. 8 refers to an office environment which is about $65 \times 20 \mathrm{~m}$. A user walks for about 3 minutes back and forth the hallways, stepping in some of the rooms; the user equipment is composed of a foot mounted IMU which provides inertial measurements and a hand-held smartphone which logs the RSS measurements from 4 APs; the WLAN standard being used is IEEE 802.11 (WiFi), version b/g; the processing has been done off-line.

In Fig. 8, panel (a), we show the extended WiSLAM, with estimation of $h_{0}$ and $\alpha$ for each AP, while in panel (b) we present the result of classic WiSLAM, both run with 5000 particles. We use 9 hypotheses for $h_{0}$ in both cases, with a $2 \mathrm{~dB}$ spacing, while only 5 values between 1.5 and $3.5(0.5$ spacing) are selected for $\alpha$ estimation in the case of panel (a). To ensure a fair comparison, we use the same computational complexity in both cases: the number of components per GMM is increased by a factor 5 in the case of classic WiSLAM version, i.e. the total number of Gaussian components per particles is the same in both cases.

The second testbed is depicted in Fig. 9 and represents another office environment about $45 \mathrm{~m}$ long and $25 \mathrm{~m}$ wide, with a circular hallway and 4 APs. The walk lasts about 7 minutes and corresponds to 3 turns in the hallway with visits to some of the offices; in this case the RSS were collected by a hand-held laptop. Fig. 9, panel (a), shows the extended WiSLAM result while panel (b) contains results with fixed $\alpha=2$ : AP 1 is well localized by both algorithms, while the main improvement is shown on AP 2; AP 3 and 4 do not show any improvement and in the case of AP 4 the resulting pdf is narrower, but centered still on the wrong position; however, the exact AP's position is still in the pdf's support.

Finally, Fig. 10 shows the bivariate pdf of the parameters, averaged over the particles, for the AP 1 of the second testbed, Fig. 9. For this result, we increased the number of hypotheses concerning the exponent, by setting $\Delta \alpha=0.125$ and the resulting discrete distribution has been smoothed by means of a Gaussian kernel to improve visualization. In panel (a), after 2 minutes of the walk and a first passage close by the AP, the bivariate pdf evolves following a line; after the second passage 


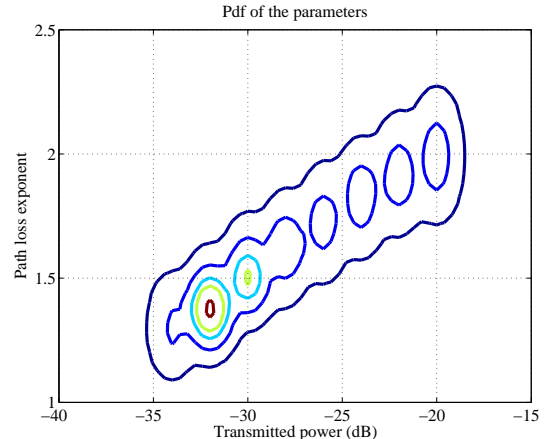

(a) Time: $120 \mathrm{~s}$

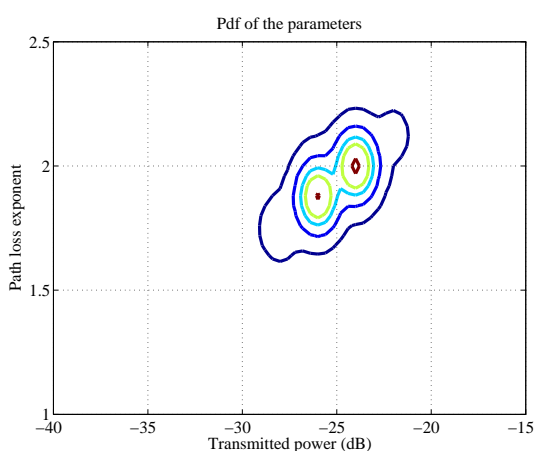

(b) Time: $200 \mathrm{~s}$

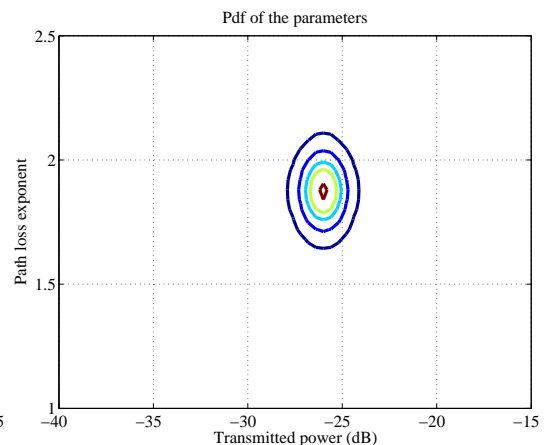

(c) Time: $420 \mathrm{~s}$

Fig. 10. Evolution of the bivariate pdf of the parameters for AP1 in the scenario of Fig. 9.

of the user in proximity of the AP (b) the pdf is narrower, since some of the hypotheses are censored and, at the end of the walk (c) only one hypothesis survives.

\section{CONCLUSION}

Path loss parameters in WLAN based SLAM are of interest since a mismatch in the model can yield relevant errors in both positioning and mapping. In this paper we discussed the issues arising from the joint estimation of transmitted power and path loss exponent. We present and discuss two main results: in the first part of the paper we dealt with only parameter estimation, by proving their joint observability in the framework of estimation theory; to show this we computed the theoretical bound of the estimator in terms of the CRLB, proving that the ML estimator is optimal according to the MMSE criterion.

In the second part of the paper we proposed an extension of WiSLAM, a WLAN-based Bayesian SLAM algorithm, which accounts also for both parameters and we showed its effectiveness in practical scenarios.

Some model mismatches are still present and in future we will consider improved path loss models, based on a sectorization of the parameters in different parts of the building. Challenging three-dimensional models, far from being mere extensions, will be developed in order to obtain a WLAN map in realistic buildings.

\section{REFERENCES}

[1] W. Kolodziej and J. Hjelm. Local positioning systems: LBS applications and services. CRC Press, 2006.

[2] S.A. Ahson and M. Mohammad Ilyas. Location-Based Services Handbook: Applications, Technologies, and Security. CRC Press, 2009.

[3] J. Rantakokko, J. Rydell, P. Stromback, P. Handel, J. Callmer, D. Tornqvist, F. Gustafsson, and M. Grudén. Accurate and reliable soldier and first responder indoor positioning: multisensor systems and cooperative localization. IEEE Wireless Commun., 18(2):10-18, 2011.

[4] B. Krach and P. Roberston. Cascaded estimation architecture for integration of foot-mounted inertial sensors. In Proc. IEEE/ION Symp. Position Location and Navigation (PLANS), pages 112 -119, May 2008.

[5] S. Beauregard, Widyawan, and M. Klepal. Indoor PDR performance enhancement using minimal map information and particle filters. In Proc. IEEE/ION Symp. Position Location and Navigation (PLANS), pages $141-147$, May 2008.
[6] Oliver Woodman and Robert Harle. Pedestrian localisation for indoor environments. In Proc. 10th Int. Conf. Ubiquitous Computing, UbiComp '08, pages 114-123, New York, NY, USA, 2008. ACM.

[7] P. Bahl and V. Padmanabhan. RADAR: An in-building RF-based user location and tracking system. Proc. IEEE INFOCOM, pages 775-784, Mar 2000.

[8] H. Durrant-Whyte and T. Bailey. Simultaneous localization and mapping: part i. IEEE Robot. Autom. Mag., 13(2), pages 99-110, june 2006.

[9] M.S. Arulampalam, S. Maskell, N. Gordon, and T. Clapp. A tutorial on particle filters for online nonlinear/non-Gaussian Bayesian tracking. IEEE Trans. On Signal Processing., 50(2), pages 174-188, Feb 2002.

[10] M. Montemerlo, S. Thrun, D. Koller, and Wegbreit B. FastSLAM: A factored solution to the simultaneous localization and mapping problem. In Proc. National Conf. Artificial Intelligence, pages 593 - 598, 2002.

[11] P. Robertson, M. Angermann, and B. Krach. Simultaneous localization and mapping for pedestrians using only foot-mounted inertial sensors. In Proceedings of the 11th international conference on Ubiquitous computing, Ubicomp '09, pages 93-96, NY, USA, 2009. ACM.

[12] M. Angermann and P. Robertson. FootsSLAM: Pedestrian simultaneous localization and mapping without exteroceptive sensors - hitchhiking on human perception and cognition. Proceedings of the IEEE, 100(Special Centennial Issue), pages 1840-1848, 132012.

[13] L. Bruno and P. Roberston. WiSLAM: improving FootSLAM with WiFi. In Proc. IEEE Int. Conf. Indoor Positioning Indoor Navigation, pages $1-10$, Sept. 2011.

[14] J. Parsons. The Mobile Radio Propagation Channel, $2^{n} d$ Ed. Wiley \& Sons, November 2000.

[15] Brian Ferris, Dieter Fox, and Neil Lawrence. WiFi-SLAM using gaussian process latent variable models. In Proc. Int. Joint Conf. Artificial Intelligence (IJCAI), pages 2480-2485, 2007.

[16] Joseph Huang, David Millman, Morgan Quigley, David Stavens, Sebastian Thrun, and Alok Aggarwal. Efficient, generalized indoor wifi graphslam. In Proc. IEEE Int. Conf. Robotics and Automation (ICRA), pages $1038-1043$, may 2011.

[17] A. Bose and Chuan Heng Foh. A practical path loss model for indoor WiFi positioning enhancement. In Information, Communications Signal Processing, 2007 6th International Conference on, pages 1-5, 2007.

[18] Qing Zhang, Chuan Heng Foh, Boon-Chong Seet, and A. C. Fong. Variable elasticity spring-relaxation: improving the accuracy of localization for WSNs with unknown path loss exponent. Personal Ubiquitous Comput., 16(7), pages 929-941, October 2012.

[19] Xinrong Li. RSS-based location estimation with unknown pathloss model. Wireless Communications, IEEE Transactions on, 5(12), pages 3626-3633, 2006.

[20] H. Nurminen, J. Talvitie, S. Ali-Loytty, P. Muller, E. Lohan, R. Piche, and M. Renfors. Statistical path loss parameter estimation and positioning using RSS measurements in indoor wireless networks. In Indoor Positioning and Indoor Navigation (IPIN), 2012 International Conference on, pages 1-9, 2012.

[21] S.M. Kay. Fundamentals of Statistical Signal Processing: Estimation Theory. Prentice Hall, 1993. 